\title{
Higroscopicidade de sementes de caju-de-árvore-do-cerrado ${ }^{1}$
}

\author{
Graciene de Souza Caetano ${ }^{2}$, Kelly Aparecida de Sousa ${ }^{2}$, \\ Osvaldo Resende², Juliana de Fátima Sales ${ }^{2}$, Lílian Moreira Costa ${ }^{2}$
}

\section{ABSTRACT}

Hygroscopicity of Anacardium othonianum Rizz. seeds

Studies on conservation and exploitation of the Brazilian Savannah native species are essential to technologies that lead to the rational exploitation of these products. This study aimed at determining the desorption isotherms of Anacardium othonianum seeds for different temperature and water activity conditions, as well as adjusting different mathematical models to the experimental data, by selecting the one that best represents the phenomenon. The hygroscopicity was determined with the aid of the gravity static method, for temperatures of $25^{\circ} \mathrm{C}, 30^{\circ} \mathrm{C}, 35^{\circ} \mathrm{C}$ and $40^{\circ} \mathrm{C}$ and water activity between 0.12 and 0.89 (decimal). It was observed that the equilibrium moisture content decreased while the temperature increased, for the same water activity, similarly to what happens with hygroscopic products. The Chung-Pfost model reached the highest determination coefficient and the lowest values for relative average error, estimated medium error and chi-square test, being the one selected to predict the hygroscopic equilibrium of Anacardium othonianum seeds. The isosteric heat increased, while the moisture content decreased, i.e., it increased the energy required to remove water, varying from $4,586.35 \mathrm{~kJ} \mathrm{~kg}^{-1}$ to $2,572.7 \mathrm{~kJ} \mathrm{~kg}^{-1}$, for the moisture content of 1.76-6.56 (d.b. \%).

KEY-WORDS: Desorption isotherms; hygroscopic equilibrium; isosteric heat of desorption; Brazilian Savannah native fruits.

\section{INTRODUÇÃO}

O Cerrado brasileiro possui flora muito abundante, com diversas espécies que merecem atenção especial, a exemplo das plantas medicinais e frutíferas (Brasil 2002, Caramori et al. 2004). Dentre estas, destaca-se, no Estado de Goiás, o caju-de-árvore-do-cerrado, árvore mediana de 3,0-6,0 m de altura e tronco de 20,0-40,0 cm de diâmetro (Naves 1999). O fruto é uma drupa reniforme (castanha), que contém cerca de 46,5\% de lipídios (Gallina et

\section{RESUMO}

Estudos sobre a conservação e exploração de espécies nativas do Cerrado são fundamentais para tecnologias que visem à exploração racional destes produtos. Objetivou-se, neste trabalho, determinar as isotermas de dessorção de sementes de caju-de-árvore-do-cerrado, para diversas condições de temperatura e atividades de água, bem como ajustar diferentes modelos matemáticos aos dados experimentais, selecionando aquele que melhor representa o fenômeno. A higroscopicidade foi determinada pelo método estático-gravimétrico, para temperaturas de $25^{\circ} \mathrm{C}, 30^{\circ} \mathrm{C}, 35^{\circ} \mathrm{C}$ e $40^{\circ} \mathrm{C}$ e atividades de água entre 0,12 e 0,89 (decimal). Observou-se que o teor de água de equilíbrio decresceu com o aumento da temperatura, para uma mesma atividade de água, à semelhança dos produtos higroscópicos. O modelo de Chung-Pfost obteve o maior coeficiente de determinação e menores valores de erro médio relativo, erro médio estimado e $Q u i$-quadrado, sendo selecionado para predição do equilíbrio higroscópico de sementes de caju-deárvore-do-cerrado. O calor isostérico aumentou com a diminuição do teor de água, ou seja, aumentou a energia necessária para a remoção de água, com valores variando de $4.586,35 \mathrm{~kJ} \mathrm{~kg}^{-1}$ a $2.572,7 \mathrm{~kJ} \mathrm{~kg}^{-1}$, na faixa de teor de água de $1,76-6,56$ (\% b.s.).

PALAVRAS-CHAVE: Isotermas de dessorção; equilíbrio higroscópico; calor isostérico de dessorção; frutas nativas do Cerrado.

al. 1993), e que pode ser consumida quando tostada (Silva et al. 2001). O pseudofruto carnoso, suculento, em forma de pêra, de coloração que varia do amarelo ao vermelho (Brandão et al. 1992), é rico em vitamina $\mathrm{C}$, fibras e compostos fenólicos (Agostini-Costa et al. 2005).

A paisagem do Cerrado tem se modificado de modo acelerado, com o desmatamento de grandes áreas, para a produção de monoculturas (Oliveira \& Rocha 2008), comprometendo a sustentabilidade do bioma e colocando muitas espécies animais e vegetais

1. Trabalho recebido em jun./2012 e aceito para publicação em dez./2012 (nºgistro: PAT 19108).

2. Instituto Federal de Educação, Ciência e Tecnologia Goiano (IF Goiano), Campus RioVerde, RioVerde, GO, Brasil. E-mails: gracienetpg@yahoo.com.br, kellyapsousa@yahoo.com.br, osvresende@yahoo.com.br, julianacefetrv@yahoo.com.br, lmctpg@yahoo.com.br. 
em risco de extinção, incluindo as fruteiras nativas (Mendonça et al. 1998).

Os frutos nativos possuem importância econômica e social. Muitos são comercializados e consumidos in natura ou beneficiados por indústrias caseiras (Silva et al. 1994), e a castanha também constitui fonte alternativa de alimento e pode ser consumida quando tostada (Vieira et al. 2006).

Os conhecimentos técnicos a respeito da propagação de uma espécie nativa são fundamentais para a definição da tecnologia de exploração racional. Neste sentido, o armazenamento de sementes constitui importante estratégia para a conservação genética ex situ de espécies vegetais, atendendo a objetivos como conservação, melhoramento ou propagação.

As condições de umidade relativa e temperatura, durante o armazenamento, promovem o equilíbrio higroscópico das sementes e determinarão a manutenção de sua qualidade fisiológica por maior ou menor tempo. A umidade relativa e a temperatura são fatores primordiais, e seu estudo tem se intensificado, ultimamente, para sementes florestais (Borges et al. 2009).

Os produtos vegetais possuem a propriedade de realizar trocas de água sob a forma de vapor, com o ambiente que os envolve. Estas trocas podem acontecer por meio do ganho ou da perda de água, fenômenos conhecidos, respectivamente, por adsorção e dessorção, de acordo com as características higroscópicas recíprocas do produto e do ar (Brooker et al. 1992), com a água sempre aumentando a pressão de vapor sobre a superfície dos produtos. Quando esta pressão e a pressão de vapor de água da atmosfera são iguais, ocorre o equilíbrio higroscópico (Araújo et al. 2005).

Para Hall (1980), as curvas de equilíbrio higroscópico são importantes para definir limites de desidratação do produto, estimar as mudanças no teor de água sob determinada condição de temperatura e umidade relativa do ambiente e definir os teores de água adequados ao início da atividade de micro-organismos que podem provocar a deterioração do produto.

A relação entre o teor de água de equilíbrio e a umidade relativa de equilíbrio, também designada por atividade da água, constitui fator essencial, em projetos e estudos de sistemas de secagem, manuseio, armazenamento, embalagem, transporte e modelagem da longevidade das sementes (Araújo et al. 2001). A relação entre o teor de água de um determinado produto e a umidade relativa de equilíbrio, para uma temperatura específica, pode ser expressa por meio de equações matemáticas denominadas isotermas, ou curvas de equilíbrio higroscópico (Corrêa et al. 2005a).

Diversos autores determinaram as isotermas de sorção para vários produtos vegetais, tais como pedúnculo seco de caju (Anacardium occidentale L.) (Alcântara et al. 2009), amido de inhame (Dioscorea alata L.) (Nunes et al. 2009, Oliveira et al. 2009), casca de maracujá (Passiflora edulis Sims) (Oliveira et al. 2006) e jacarandá-da-bahia (Dalbergia nigra Fr. All.), angico-vermelho (Anadenanthera macrocarpa Benth.) e óleo-copaíba (Copaifera langsdorffii Benth.) (Mesquita et al. 2001).

$\mathrm{O}$ conhecimento das isotermas de sorção de água e do calor isostérico líquido de sorção é de grande importância para os processos de secagem, armazenamento e embalagem. Eles são úteis para calcular o tempo e a energia consumidos durante a secagem, para, prevendo-se o comportamento do produto, auxiliar na seleção da embalagem, modelar variações de teor de água durante o armazenamento e estimar a vida de prateleira dos produtos alimentícios (Siripatrawan \& Jantawat 2006, Catelam et al. 2011, Comunian et al. 2011). Estas propriedades também fornecem informações sobre os mecanismos de sorção e interações entre os componentes químicos dos produtos e a água (Gabas et al. 2000).

Diante da importância do conhecimento da higroscopicidade dos produtos vegetais, objetivou-se, neste trabalho, determinar as isotermas de dessorção de sementes de caju-de-árvore-do-cerrado, para diversas condições de temperatura e atividades de água, bem como ajustar diferentes modelos matemáticos aos dados experimentais, selecionando aquele que melhor representa o fenômeno e, assim, utilizá-lo para determinar o calor isostérico de dessorção.

\section{MATERIAL E MÉTODOS}

O experimento foi desenvolvido no Laboratório de Pós-Colheita de Produtos Vegetais do Instituto Federal de Educação, Ciência e Tecnologia Goiano (IF Goiano), Campus Rio Verde (GO).

Para a realização deste trabalho, foram utilizadas sementes de caju-de-árvore-do-cerrado colhidas de cinco plantas matrizes, em setembro de 2010, na Fazenda Gameleira, Município de Montes Claros de Goiás (GO). Após a coleta manual dos frutos, realizou-se a despolpa manual, sendo removido o 
pseudofruto (pedúnculo) e utilizada apenas a amêndoa, quando verificou-se que o teor de água inicial, determinado pelo método da estufa, a $105 \pm 3^{\circ} \mathrm{C}$ (Brasil 2009), foi de 17,54\% (b.s.).

Para obtenção do teor de água de equilíbrio higroscópico de sementes de caju-de-árvore-do-cerrado, foi utilizado o método estático-gravimétrico, de acordo com Hall (1957), com adaptações. A dessorção do produto, em camada delgada de, aproximadamente, $5,0 \mathrm{~cm}$, foi realizada para diferentes condições controladas de temperatura $\left(25^{\circ} \mathrm{C}, 30^{\circ} \mathrm{C}\right.$, $35^{\circ} \mathrm{C} \mathrm{e} 40^{\circ} \mathrm{C}$ ), fornecidas por uma câmara tipo BOD, e atividades de água entre 0,$12 ; 0,15 ; 0,22 ; 0,30 ; 0,44$; 0,$45 ; 0,46 ; 0,76 ; 0,75 ; 0,79 ; 0,81$; e 0,89 (decimal), até que o produto atingisse seu teor de água de equilíbrio, com a condição do ar especificada.

As amostras, contendo cada uma 10,0 g, correspondentes a, aproximadamente, cinco sementes de caju-de-árvore-do-cerrado, foram envolvidas por um tecido permeável (tipo voile), para permitir a troca de vapor de água, e mantidas no interior de dessecadores contendo soluções salinas saturadas.

As soluções salinas utilizadas para gerar ambientes de diferentes atividades de água foram as soluções com cloreto de lítio $(\mathrm{LiCl})$, cloreto de cálcio $\left(\mathrm{CaCl}_{2}\right)$, nitrato de cálcio $\left(\mathrm{Ca}\left(\mathrm{NO}_{3}\right)_{2}\right)$, cloreto de sódio $(\mathrm{NaCl})$ e brometo de potássio (KBr) (Tabela 1). Durante o processo de dessorção, as amostras foram pesadas, periodicamente, até atingirem o equilíbrio higroscópio, ou seja, até que não houvesse variação de massa, após três pesagens consecutivas, e, em seguida, foram determinados os teores de água (Brasil 2009).

Os dados experimentais do teor de água de equilíbrio foram ajustados aos modelos matemáticos apresentados na Tabela 2, tradicionalmente utilizados para predizer a higroscopicidade de produtos vegetais.

Tabela 1. Atividades de água (decimal) estabelecidas no interior dos dessecadores, para a determinação do equilíbrio higroscópico de sementes de caju-de-árvore-do-cerrado (Rio Verde, GO, 2011).

\begin{tabular}{|c|c|c|c|c|}
\hline \multirow{2}{*}{ Sal } & \multicolumn{4}{|c|}{ Temperatura $\left({ }^{\circ} \mathrm{C}\right)$} \\
\hline & 25 & 30 & 35 & 40 \\
\hline $\mathrm{LiCl}$ & 0,120 & 0,151 & 0,149 & - \\
\hline $\mathrm{CaCl}_{2}$ & 0,303 & 0,279 & 0,217 & - \\
\hline $\mathrm{Ca}\left(\mathrm{NO}_{3}\right)_{2}$ & - & 0,462 & 0,441 & 0,450 \\
\hline $\mathrm{NaCl}$ & 0,740 & - & - & 0,746 \\
\hline $\mathrm{KBr}$ & 0,814 & - & - & 0,791 \\
\hline
\end{tabular}

Tabela 2. Modelos matemáticos utilizados para predizer a higroscopicidade de sementes de caju-de-árvoredo-cerrado.

\begin{tabular}{ll}
\hline \multicolumn{1}{c}{ Designação do modelo } & \multicolumn{1}{c}{ Modelo } \\
\hline $\mathrm{Xe}=\exp \left\{a-(b \cdot T)+\left[c \cdot \exp \left(a_{w}\right)\right]\right\}$ & Sigma Copace \\
$\mathrm{Xe}=\mathrm{a} \cdot\left(\mathrm{a}_{\mathrm{w}} \mathrm{b} / \mathrm{T}^{\mathrm{c}}\right)$ & Sabbah \\
$\mathrm{Xe}=(\mathrm{a}+\mathrm{bT}) /\left[\left(1-\mathrm{a}_{\mathrm{w}}\right) / \mathrm{a}_{\mathrm{w}}\right]^{1 / \mathrm{c}}$ & Oswin \\
$\mathrm{Xe}=\left[\left(\mathrm{a} \cdot \mathrm{a}_{\mathrm{w}}{ }^{\mathrm{b}}\right)+\left(\mathrm{c} \cdot \mathrm{a}_{\mathrm{w}}{ }^{\mathrm{d}}\right)\right]$ & Peleg \\
$\mathrm{Xe}=\left[\exp (\mathrm{a}-\mathrm{b} \cdot \mathrm{T}) /-\operatorname{In}\left(\mathrm{a}_{\mathrm{w}}\right)\right]^{1 / \mathrm{c}}$ & Halsey \\
$\mathrm{Xe}=\left(\mathrm{a} \cdot \mathrm{b} \cdot \mathrm{c} \cdot \mathrm{a}_{\mathrm{w}}\right) /\left[\left(1-\mathrm{c} \cdot \mathrm{a}_{\mathrm{w}}\right) \cdot\left(1-\mathrm{c} \cdot \mathrm{a}_{\mathrm{w}}+\mathrm{b} \cdot \mathrm{c} \cdot \mathrm{a}_{\mathrm{w}}\right)\right]$ & GAB \\
$\mathrm{Xe}=\exp \left[\mathrm{a}-(\mathrm{b} \cdot \mathrm{T})+\left(\mathrm{c} \cdot \mathrm{a}_{\mathrm{w}}\right)\right]$ & Copace \\
$\mathrm{Xe}=\mathrm{a}-\mathrm{b} \cdot \operatorname{In}\left[-(\mathrm{T}+\mathrm{c}) \cdot \operatorname{In}\left(\mathrm{a}_{\mathrm{w}}\right)\right]$ & Chung-Pfost \\
$\mathrm{Xe}=\left[\left(\mathrm{a} \cdot \mathrm{b} \cdot \mathrm{a}_{\mathrm{w}}\right) /\left(1-\left(\mathrm{c} \cdot \mathrm{a}_{\mathrm{w}}\right) \cdot\left(1+(\mathrm{b}-\mathrm{c}) \cdot \mathrm{a}_{\mathrm{w}}\right)\right]\right.$ & BET \\
$\mathrm{Xe}=\mathrm{a}-(\mathrm{b} \cdot \mathrm{T})-\mathrm{c} \cdot \ln \left(1-\mathrm{a}_{\mathrm{w}}\right)$ & Modificado \\
\hline
\end{tabular}

$\mathrm{Xe}=$ teor de água de equilíbrio (\% b.s.); $\mathrm{a}_{\mathrm{w}}=$ atividade de água (decimal); $\mathrm{T}=$ temperatura $\left({ }^{\circ} \mathrm{C}\right) ; \mathrm{a}, \mathrm{b}, \mathrm{c}, \mathrm{d}=$ coeficientes que dependem do produto.

Para o ajuste dos modelos matemáticos, foi realizada análise de regressão não linear, pelo método Gauss Newton, utilizando-se o programa computacional Statistica $7.0^{\circledR}$. Como critério de seleção, para o modelo que melhor representasse o processo, foi considerada a significância do coeficiente de regressão, pelo teste t, adotando-se o nível de 5\% de significância, magnitude do coeficiente de determinação $\left(\mathrm{R}^{2}\right)$, valores do erro médio relativo $(\mathrm{P})$ e do erro médio estimado (SE) e teste de Qui-quadrado $\left(\chi^{2}\right)$, com intervalo de confiança a $95 \%(\mathrm{p}<0,05)$. Considerou-se o valor do erro médio relativo inferior a $10 \%$ como um dos critérios para a seleção dos modelos, de acordo com Mohapatra \& Rao (2005). Os erros médios relativos e estimados e o teste de Qui-quadrado, para cada um dos modelos, foram calculados, respectivamente, conforme as seguintes equações:

$P=\frac{100}{n} \sum \frac{|Y-\hat{Y}|}{Y}, \quad S E=\sqrt{\frac{\sum(Y-\hat{Y})^{2}}{G L R}}, \quad \chi^{2}=\sum \frac{(\mathrm{Y}-\hat{Y})^{2}}{G L R}$,

em que $Y=$ valor observado experimentalmente; $\hat{Y}=$ valor calculado pelo modelo; $n=$ número de observações experimentais; e $G L R=$ graus de liberdade do modelo (número de observações menos o número de parâmetros do modelo). 
Para os cálculos do calor isostérico líquido de sorção (ou entalpia diferencial), para cada teor de água de equilíbrio, foi utilizada a equação de Clausius-Clayperon (Iglesias \& Chirife 1976):

$$
\frac{\partial \operatorname{In}\left(\mathrm{a}_{\mathrm{w}}\right)}{\partial \mathrm{T}}=\frac{\Delta \mathrm{h}_{\mathrm{st}}}{\mathrm{RT}_{\mathrm{a}}^{2}},
$$

em que $\mathrm{a}_{\mathrm{w}}=$ atividade de água (decimal); $\mathrm{T}_{\mathrm{a}}=$ temperatura absoluta $(\mathrm{K}) ; \Delta \mathrm{h}_{\mathrm{st}}=$ entalpia diferencial $\left(\mathrm{kJ} \mathrm{kg}^{-1}\right) ; \mathrm{R}=$ constante universal dos gases $\left(8,314 \mathrm{~kJ} \mathrm{kmol}^{-1} \mathrm{~K}^{-1}\right)$, sendo, para o vapor d'água, de $0,4619 \mathrm{~kJ} \mathrm{~kg}^{-1} \mathrm{~K}^{-1}$.

Integrando-se a equação de Clausius-Clayperon e assumindo-se que o calor isostérico líquido de sorção é independente da temperatura, pode-se encontrar o calor isostérico líquido de sorção, para cada teor de água de equilíbrio, conforme a seguinte equação (Wang \& Brennan 1991):

$$
\operatorname{In}\left(a_{w}\right)=-\left(\frac{\Delta h_{s t}}{R}\right) \cdot \frac{1}{T_{a}}+C,
$$

em que $\mathrm{C}=$ coeficiente do modelo.

O calor isostérico integral de dessorção foi obtido adicionando-se aos valores de calor isostérico líquido de sorção o valor do calor latente de vaporização da água livre, de acordo com a equação:

$$
\mathrm{Q}_{\mathrm{st}}=\Delta \mathrm{h}_{\mathrm{st}}+\mathrm{L}=\mathrm{a} \cdot \exp (-\mathrm{b} \cdot \mathrm{Xe})+\mathrm{c},
$$

em que $\mathrm{Q}_{\mathrm{st}}=$ calor isostérico integral de sorção $\left(\mathrm{kJ} \mathrm{kg}^{-1}\right) ; \mathrm{a}, \mathrm{b}, \mathrm{c}=$ coeficientes do modelo.

$\mathrm{O}$ calor latente de vaporização da água livre (L) $\left(\mathrm{kJ} \mathrm{kg}^{-1}\right)$ necessário ao cálculo de $\mathrm{Q}_{\mathrm{st}}$ foi obtido utilizando-se a temperatura média $(\mathrm{T})\left({ }^{\circ} \mathrm{C}\right)$ na faixa em estudo, por meio da seguinte equação:

$$
\mathrm{L}=2502,2-2,39 . \mathrm{T} \text {, }
$$

em que $\mathrm{L}=$ calor latente de vaporização da água livre $\left(\mathrm{kJ} \mathrm{kg}^{-1}\right)$.

\section{RESULTADOS E DISCUSSÃO}

Os valores médios do teor de água de equilíbrio higroscópico das sementes de caju-de-árvore-do-cerrado, obtidos por dessorção, para as temperaturas de $25^{\circ} \mathrm{C}, 30^{\circ} \mathrm{C}, 35^{\circ} \mathrm{C}$ e $40^{\circ} \mathrm{C}$ e diferentes atividades de água, estão apresentados na Tabela 3 .

Observa-se que, para uma mesma temperatura, com o incremento da atividade de água, tem-se aumento no teor de água de equilíbrio, para as sementes de caju-de-árvore-do-cerrado (Tabela 3). Resultados semelhantes foram encontrados por Gomes et al. (2002), em polpa de acerola (Malphighiae marginata D. C.) em pó, às temperaturas de $20^{\circ} \mathrm{C}$, $25^{\circ} \mathrm{C}$ e $30^{\circ} \mathrm{C}$; Alexandre et al. (2007), em pitanga (Eugenia uniflora L.) em pó, às temperaturas de $10^{\circ} \mathrm{C}, 20^{\circ} \mathrm{C}, 30^{\circ} \mathrm{C}$ e $40^{\circ} \mathrm{C}$; e Oliveira et al. (2011), para amêndoa de cacau (Theobroma cacao), às temperaturas de $25^{\circ} \mathrm{C}, 35^{\circ} \mathrm{C}, 45^{\circ} \mathrm{C}$ e $55^{\circ} \mathrm{C}$.

$\mathrm{Na}$ Tabela 4, estão apresentados os parâmetros dos modelos ajustados aos valores de teor de água de equilíbrio higroscópico, para as sementes de caju-de-árvore-do-cerrado, obtidos por dessorção, para diferentes condições de temperatura e atividade de água.

Observou-se que os modelos matemáticos utilizados para descrever a higroscopicidade das sementes de caju-de-árvore-do-cerrado apresentaram, para a maioria dos seus coeficientes, significância de regressão de $1 \%$, pelo teste t (Tabela 4).

Os modelos Sabbah, Pelleg, Copace, Chung-Pfost e BET Modificado exibiram elevados valores de coeficiente de determinação (superiores a 96\%), fato que, de acordo com Madamba et al. (1996), indica uma representação satisfatória do fenômeno em estudo. Segundo estes pesquisadores, a utilização do coeficiente de determinação como o único critério de avaliação para a seleção dos modelos não lineares não constitui um bom parâmetro para representação do fenômeno em estudo. Para uma análise mais detalhada, utilizaram-se outros parâmetros estatísticos, para respaldar a seleção do melhor modelo.

Tabela 3. Valores médios do teor de água de equilíbrio (\% b.s.) de sementes de caju-de-árvore-do-cerrado obtidos por dessorção, em função da temperatura $\left({ }^{\circ} \mathrm{C}\right)$ e da atividade de água (decimal, $\left.\pm 0,03\right)$ (Rio Verde, GO, 2012).

\begin{tabular}{ccccccccccccc}
\hline $\begin{array}{c}\text { Temperatura } \\
\left({ }^{\circ} \mathrm{C}\right)\end{array}$ & 0,12 & 0,15 & 0,22 & 0,30 & 0,44 & 0,45 & 0,46 & 0,74 & 0,75 & 0,79 & 0,81 & 0,89 \\
\hline & \multicolumn{1}{c}{2,35} & - & - & 3,50 & - & - & - & 5,80 & - & - & 6,46 & - \\
30 & - & 1,76 & - & - & - & - & 3,87 & 5,75 & - & - & - & 6,57 \\
35 & - & 1,73 & 2,35 & - & 3,50 & - & - & - & - & - & - & - \\
40 & - & - & - & - & - & 3,26 & - & - & 5,62 & 5,89 & - & - \\
\hline
\end{tabular}


Tabela 4. Coeficientes dos modelos ajustados aos teores de água de equilíbrio higroscópico para as sementes de caju-de-árvore-docerrado, com seus respectivos coeficientes de determinação $\left(\mathrm{R}^{2}\right)$, erros médios relativos $(\mathrm{P})$, erros médios estimados $(\mathrm{SE})$ e Qui-quadrado $\left(\chi^{2}\right)$ (Rio Verde, GO, 2012).

\begin{tabular}{|c|c|c|c|c|c|}
\hline Modelos & Coeficientes & $\mathrm{R}^{2}(\%)$ & $\mathrm{P}(\%)$ & SE (decimal) & $\chi^{2}$ \\
\hline Sigma Copace & $\begin{array}{l}\mathrm{a}=0,083041^{\mathrm{ns}} \\
\mathrm{b}=0,004445^{* *} \\
\mathrm{c}=0,834361^{* *}\end{array}$ & 94,20 & 11,182 & 0,701 & 0,202 \\
\hline Sabbah & $\begin{array}{l}\mathrm{a}=17,41643^{* *} \\
\mathrm{~b}=0,67726^{* *} \\
\mathrm{c}=0,26640^{* *}\end{array}$ & 96,79 & 7,352 & 0,388 & 0,112 \\
\hline Pelleg & $\begin{array}{l}\mathrm{a}=3,457269^{*} \\
\mathrm{~b}=2,111852^{*} \\
\mathrm{c}=4,258419^{*} \\
\mathrm{~d}=0,381012^{*}\end{array}$ & 96,51 & 8,820 & 0,440 & 0,133 \\
\hline Smith & $\begin{array}{l}\mathrm{a}=2,463693 * * \\
\mathrm{~b}=2,030540 * *\end{array}$ & 92,71 & 11,423 & 0,846 & 0,235 \\
\hline Halsey Modificado & $\begin{array}{l}\mathrm{a}=3,379067^{* *} \\
\mathrm{~b}=0,010026^{\mathrm{ns}} \\
\mathrm{c}=2,581460^{* *}\end{array}$ & 91,06 & 12,965 & 1,080 & 0,3118 \\
\hline GAB & $\begin{array}{l}\mathrm{a}=4,537048^{* *} \\
\mathrm{~b}=-0,019281^{*} \\
\mathrm{c}=3,363186^{* *}\end{array}$ & 94,60 & 8,992 & 0,447 & 0,129 \\
\hline Copace & $\begin{array}{l}\mathrm{a}=0,797808^{* *} \\
\mathrm{~b}=0,005577^{n \mathrm{~ns}} \\
\mathrm{c}=1,479701^{* *}\end{array}$ & 96,50 & 8,374 & 0,423 & 0,122 \\
\hline Chung-Pfost & $\begin{array}{l}a=10,21071^{* *} \\
b=1,79224^{* *} \\
c=15,18570^{*}\end{array}$ & 96,87 & 6,792 & 0,378 & 0,109 \\
\hline BET mod. & $\begin{array}{l}\mathrm{a}=3,325754^{* *} \\
\mathrm{~b}=8,040258^{\mathrm{ns}} \\
\mathrm{c}=0,612016^{* *}\end{array}$ & 96,30 & 8,992 & 0,447 & 0,129 \\
\hline Oswin & $\begin{array}{l}\mathrm{a}=4,537048^{* *} \\
\mathrm{~b}=-0,019281^{*} \\
\mathrm{c}=3,363186^{* *}\end{array}$ & 94,59 & 10,162 & 0,654 & 0,174 \\
\hline
\end{tabular}

$* *,{ }^{*} \mathrm{e}^{\text {ns: }}$ : Significativo a $1 \%$ e $5 \%$ e não significativo, respectivamente, pelo teste t.

De acordo com os valores obtidos para erro médio estimado (SE), concluiu-se que os modelos Sabbah, Copace e Chung-Pfost apresentaram menores valores, quando comparados aos demais. Observou-se, ainda, que os valores de erro médio relativo (P) foram inferiores a 10\%, exceto para os modelos de Sigma Copace, Smith, Halsey Modificado e Oswin, fato que, segundo Mohapatra \& Rao (2005), sugere uma representação adequada do fenômeno estudado.

Em relação ao teste de Qui-quadrado $\left(\chi^{2}\right)$, os dez modelos analisados encontram-se no intervalo de confiança de 95\%. Comparando-se os modelos, observou-se que os de Sabbah e Chung-Pfost foram os que melhor se ajustaram aos dados experimentais, sendo, assim, sugeridos para descrever o fenômeno de higroscopicidade de sementes de caju-de-árvore- -do-cerrado. Considerando-se estes dois modelos, verificou-se que o de Chung-Pfost exibiu maior coeficiente de determinação $\left(\mathrm{R}^{2}\right)$, menores valores de erro médio relativo $(\mathrm{P})$ e erro médio estimado $(\mathrm{SE})$ e menor magnitude dos valores de Qui-quadrado $\left(\chi^{2}\right)$, sendo, desta maneira, o mais recomendado para a predição do equilíbrio higroscópico de sementes de caju-de-árvore-do-cerrado.

Na Figura 1, estão apresentados os valores experimentais do teor de água de equilíbrio de sementes de caju-de-árvore-do-cerrado, obtidos por dessorção, bem como suas isotermas, estimadas pelo modelo de Chung-Pfost.

Verificou-se que, para uma atividade de água constante, os valores do teor de água de equilíbrio higroscópico das sementes de caju-de-árvore-do- 


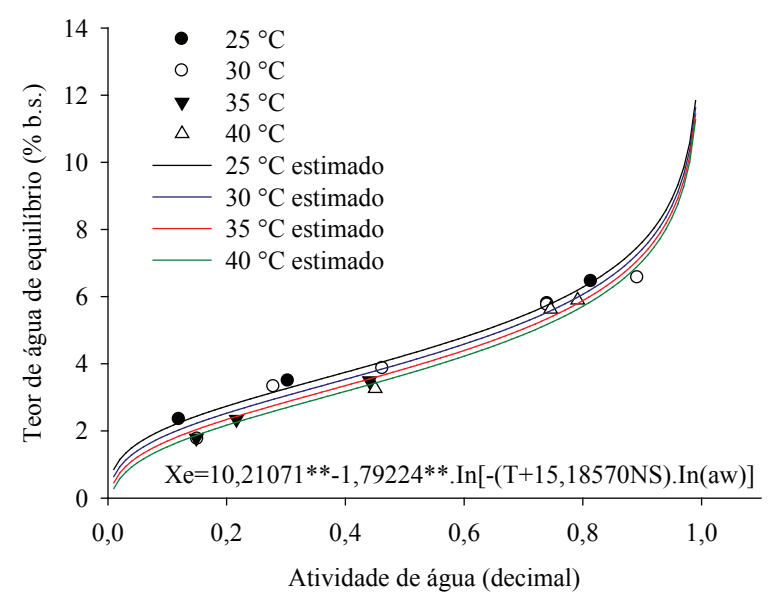

Figura 1. Valores experimentais do teor de água de equilíbrio higroscópico e isotermas de dessorção estimadas pelo modelo de Chung-Pfost, para sementes de cajude-árvore-do-cerrado, em diferentes condições de temperatura e atividades de água (Rio Verde, GO, 2012). ** Significativo a $1 \%$, pelo teste $\mathrm{t}$; ${ }^{\text {ns }}$ não significativo.

cerrado diminuíram com o aumento da temperatura (Figura 1), seguindo a mesma tendência da maioria dos produtos vegetais já estudados, como observado para isotermas de dessorção de tâmaras (Phoenix dactylifera) (Prado et al. 1999), polpa de manga (Mangifera indica L.) (Silva et al. 2002), farinha de pupunha (Bactrisgasipaes kunth) (Ferreira \& Pena 2003), milho (Zea mays L.) (Corrêa et al. 2005a), mamona (Ricinus communis L.) (Goneli 2008) e sementes de quiabo (Abelmoschus esculentus L.) (Goneli et al. 2010).

As isotermas de dessorção das sementes de caju-de-árvore-do-cerrado são curvas do tipo II (Figura 1), seguindo-se a classificação de Brunauer (Brunauer et al. 1938), cuja forma sigmoidal é utilizada, principalmente, em isotermas de produtos orgânicos.

Nunes et al. (2009), estudando o processo de sorção de água para o amido de inhame, verificaram que o modelo de Chung-Pfost foi o que melhor se ajustou aos seus dados experimentais. Outros autores recomendam este modelo para descrição da higroscopicidade de arroz (Oryza sativa) em casca (Basunia \& Abe 2001), milho doce (Zea mays L.) (Araújo et al. 2001), trigo (Triticum spp.) (Corrêa et al. 2005b), alpiste (Phalaris canariensis L.) e painço (Setaria italica) (Corrêa et al. 2006), folha de louro (Lauro nobilis L.) (Silva \& Devilla 2005) e sementes de quiabo (Abelmoschus esculentus L.) (Goneli et al. 2010).
Na Figura 2, são apresentadas as curvas do logaritmo neperiano da atividade de água (decimal), calculadas a partir da equação de Chung-Pfost, para específicos valores de teor de água de equilíbrio ( $\%$ b.s.), em função de valores do inverso da temperatura absoluta $(\mathrm{K})$, para sementes de caju-de-árvore-do-cerrado.

Os valores de entalpia diferencial $\left(\Delta \mathrm{h}_{\mathrm{st}}\right)$ $\left(\mathrm{kJ} \mathrm{kg}^{-1}\right)$, para as sementes de caju-de-árvore-do-cerrado, em função do teor de água de equilíbrio (\% b.s.), foram calculados de acordo com a equação descrita por Wang \& Brennan (1991) e representados, para cada situação, pelas inclinações das retas ilustradas na Figura 2.

Para o calor isostérico integral de dessorção $\left(\mathrm{Q}_{\mathrm{st}}\right)\left(\mathrm{kJ} \mathrm{kg}^{-1}\right)$, foram adicionados, aos valores de $\Delta \mathrm{h}_{\mathrm{st}}$, o valor do calor latente de vaporização da água livre (L'), que representa a mínima quantidade de energia necessária para evaporar a água, calculado para a temperatura média de $32,5^{\circ} \mathrm{C}$, resultando no valor de $2.424,52 \mathrm{~kJ} \mathrm{~kg}^{-1}$.

Na Figura 3, são apresentados os valores do calor isostérico integral de dessorção $\left(\mathrm{Q}_{\mathrm{s}}\right)$, em função do teor de água de equilíbrio (\% b.s.).

Observou-se que, com a redução no teor de água, ocorreu aumento da energia necessária para a remoção de água do produto, representada pelos valores do calor isostérico integral de dessorção $\left(\mathrm{Q}_{\mathrm{st}}\right)$ (Figura 3), conforme observado para diversos produtos alimentícios, como folha e caule de coentro desidratados (Coriandrum sativum L.) (Silva et al. 2010) e polpa de maracujá desidratado

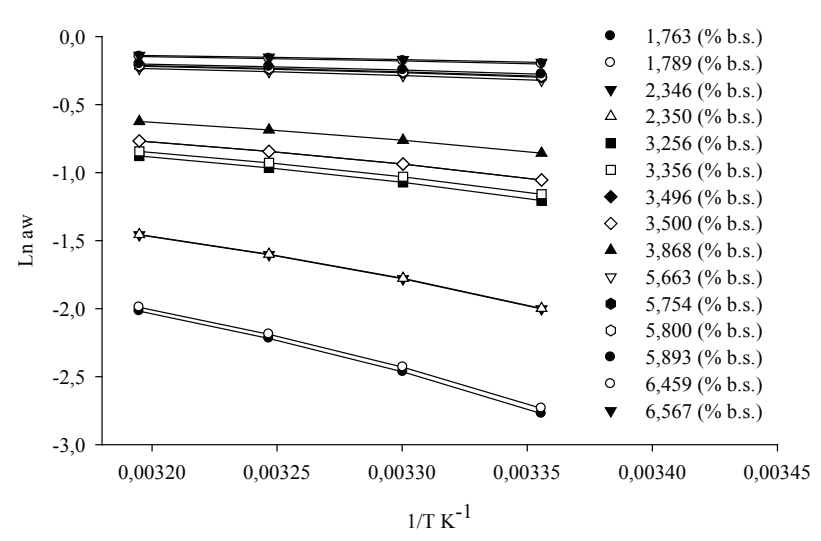

Figura 2. Curvas do logaritmo neperiano da atividade de água (decimal), para específicos valores de teor de água de equilíbrio (\% b.s.), em função de valores de $1 / \mathrm{T}\left(\mathrm{K}^{-1}\right)$, para sementes de caju-de-de-árvore-do-cerrado (Rio Verde, GO, 2012). 


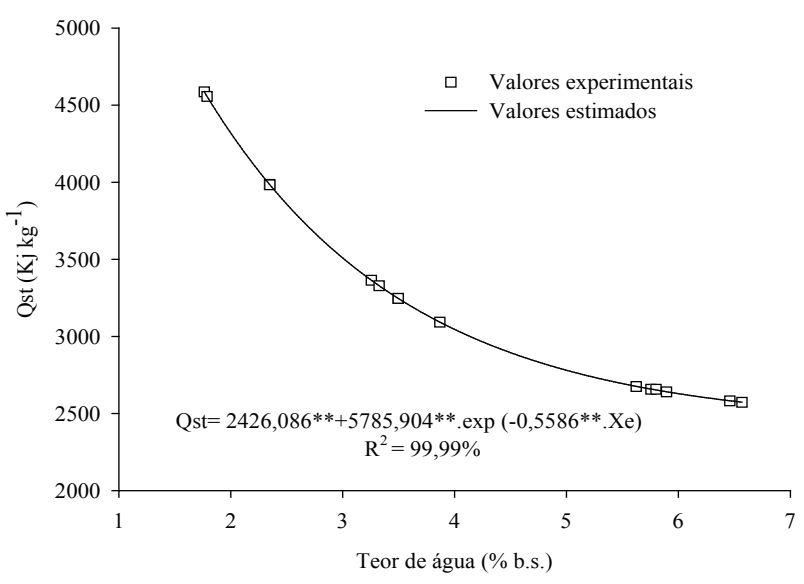

Figura 3. Valores experimentais e estimados do calor isostérico integral de dessorção, em função do teor de água de equilíbrio, para sementes de caju-de-árvore-do-cerrado (Rio Verde, GO, 2012). ** Significativo a $1 \%$, pelo teste $\mathrm{t}$.

(Passiflora edulis fo. Alavicarpa O. Deg) (Catelam et al. 2011).

Brooker et al. (1992) afirmam que, para retirar água de sementes com baixo teor de água, é necessária uma quantidade de energia superior àquela exigida para produtos úmidos. Segundo Aviara \& Ajibola (2002), estes resultados confirmam o fato de que, em produtos com maior teor de água, a força de ligação entre as moléculas de água e a matéria seca diminui sensivelmente.

\section{CONCLUSÕES}

1. As isotermas obtidas em sementes de caju-deárvore-do-cerrado possuem formato sigmoidal, padrão para o equilíbrio higroscópico de produtos agrícolas.

2. O teor de água de equilíbrio higroscópico de sementes de caju-de-árvore-do-cerrado foi diretamente proporcional à atividade de água e decresceu com o aumento da temperatura, para um mesmo valor de atividade de água.

3. O modelo de Chung-Pfost foi o que melhor representou a higroscopicidade das sementes de caju-de-árvore-do-cerrado.

4. Os valores de calor isostérico integral de dessorção, para sementes de caju-de-árvore-do-cerrado, variaram de $4.586,35 \mathrm{~kJ} \mathrm{~kg}^{-1}$ a $2.572,70 \mathrm{~kJ} \mathrm{~kg}^{-1}$, na faixa de teor de água de 1,76-6,56 (\% b.s.).

\section{REFERENNCIAS}

AGOSTINI-COSTA, T. S.; VIEIRA, R. F.; NAVES, R. V. Caju: identidade tropical que exala saúde. 2005. Disponível em: <http://www.embrapa.br/imprensa/ artigos/2005/artigo.2005-12-29.6574944222>. Acesso em: 08 fev. 2012.

ALCÂNTARA, S. R. et al. Isotermas de adsorção do pedúnculo seco do caju. Revista Brasileira de Engenharia Agrícola e Ambiental, Campina Grande, v. 13, n. 1, p. 8187, 2009.

ALEXANDRE, H. V.; FIGUEIREDO, R. M. F.; QUEIROZ, A. J. M. Isotermas de adsorção de umidade da pitanga em pó. Revista de Biologia e Ciências da Terra, Campina Grande, v. 7, n. 1, p. 11-20, 2007.

ARAÚJO, E. F.; CORRÊA, P. C.; SILVA, R. F. Comparação de modelos matemáticos para descrição das curvas de dessorção de sementes de milho doce. Pesquisa Agropecuária Brasileira, Brasília, DF, v. 36, n. 7, p. 991995, 2001.

ARAÚJO, L. F. et al. Equilíbrio higroscópico da palma forrageira: relação com a umidade ótima para fermentação sólida. Revista Brasileira de Engenharia Agrícola e Ambiental, Campina Grande, v. 9, n. 3, p. 379-384, 2005.

AVIARA, N. A.; AJIBOLA, O. O. Thermodynamics of moisture sorption in melon seed and cassava. Journal of Food Engineering, London, v. 55, n. 2, p. 107-113, 2002.

BASUNIA, M. A.; ABE, T. Moisture desorption isotherms of medium-grain rough rice. Journal of Stored Products Research, Oxford, v. 37, n. 3, p. 205-219, 2001.

BORGES, S. et al. Equilíbrio higroscópico e viabilidade de sementes de angico-vermelho (Anadenanthera peregrina (L.) Speng) em diferentes condições ambientais de armazenamento. Scientia Florestalis, Piracicaba, v. 37, n. 84, p. 475-481, 2009.

BRANDÃO, M.; CARVALHO, P. G. S.; JESUÉ, G. Guia ilustrado de plantas do Cerrado de Minas Gerais. Belo Horizonte: Cemig, 1992.

BRASIL. Ministério da Agricultura e Reforma Agrária. Secretaria Nacional de Defesa Agropecuária. Regras para análise de sementes. Brasília, DF: MARA, 2009.

BRASIL. Ministério da Agricultura, Pecuária e Abastecimento. Frutas nativas do Cerrado brasileiro: aproveitamento alimentar. Brasília, DF: Embrapa, 2002.

BROOKER, D. B.; BAKKER-ARKEMA, F. W.; HALL, C. W. Drying and storage of grains and oilseeds. Westport: The AVI Publishing Company, 1992.

BRUNAUER, S.; EMMET, P.; TELLER, E. Adsorption of gases in multimolecular layers. Journal of the American 
Chemical Society, Washington, DC, v. 60, n. 2, p. 309319, 1938.

CARAMORI, S. S.; LIMA, C. S.; FERNANDES, K. F. Biochemical characterization of selected plant species from Brazilian Savannas. Brazilian Archives of Biology and Technology, Curitiba, v. 47, n. 2, p. 253-259, 2004.

CATELAM, K. T.; TRINDADE, C. S. F.; ROMERO, J. T. Isotermas de adsorção e calor isostérico de sorção de polpa de maracujá desidratada por spray dryer e liofilizador com aditivos e leite desnatado. Ciência e Agrotecnologia, Lavras, v. 35, n. 6, p. 1196-1203, 2011.

COMUNIAN, T. A. et al. Assessment of production efficiency, physicochemical properties and storage stability of spray-dried chlorophyllide, a natural food colourant, using gum Arabic, maltodextrin and soy protein isolatebased carrier systems. International Journal of Food Science and Technology, Oxford, v. 46, n. 6, p. 12591265, 2011.

CORREAA, P. C.; RESENDE, O.; RIBEIRO, D. M. Isotermas de sorção das espigas de milho: obtenção e modelagem. Revista Brasileira de Milho e Sorgo, Sete Lagoas, v. 4, n. 1, p. 126-134, 2005 a.

CORRÊA, P. C. et al. Obtenção e modelagem das isotermas de dessorção e do calor isostérico de dessorção para grãos de trigo. Revista Brasileira de Produtos Agroindustriais, Campina Grande, v. 7, n. 1, p. 39-48, 2005 b.

CORRÊA, P. C. et al. Equilíbrio higroscópico de milheto, alpiste e painço: obtenção e modelagem. Revista Brasileira de Engenharia Agrícola e Ambiental, Campina Grande, v. 10, n. 1, p. 162-167, 2006.

FERREIRA, C. D.; PENA, R. S. Comportamento higroscópico da farinha de pupunha (Bactris gasipaes). Ciência e Tecnologia de Alimentos, Campinas, v. 23, n. 2, p. 251-255, 2003.

GABAS, A. L.; MENEGALLI, F. C.; TELISROMERO, J. Water sorption enthalpy-entropy compensation based on isotherms of plum skin and pulp. Journal of Food Science, Chicago, v. 65, n. 4, p. 680-684, 2000.

GALLINA, T. et al. A study on cashew nut oil composition. Journal of the American Oil Chemist's Society, Champaign, v. 70, n. 10, p. 1017-1020, 1993.

GOMES, P. M. A.; FIGUEIREDO, R. M. F.; QUEIROZ, A. J. M. Caracterização e isotermas de adsorção de umidade da polpa de acerola em pó. Revista Brasileira de Produtos Agroindustriais, Campina Grande, v. 4, n. 2 , p. 157-165, 2002.

GONELI, A. L. D. Variação das propriedades fisícomecânicas e da qualidade de mamona (Ricinus communis L.) durante a secagem e o armazenamento. 2008. $186 \mathrm{f}$. Tese (Doutorado em Engenharia Agrícola) - Universidade Federal de Viçosa, Viçosa, 2008.
GONELI, A. L. D. et al. Water desorption and thermodynamic properties of okra seeds. Transactions of the ASABE, St. Joseph, v. 53, n. 1, p. 191-197, 2010.

HALL, C. W. Drying and storage of agricultural crops. Westport: The AVI Publishing Company, 1980.

HALL, C. W. Drying farm crops. Ann Arbor: Edwardf, 1957.

IGLESIAS, H.; CHIRIFE, J. Isosteric heats of water vapour sorption on dehydrated foods. Part II: hysteresis and heat of sorption comparison with BET theory. Lebensmittel Wissenschaft and Technologie, Zürich, v. 9, n. 1, p. 123-127, 1976.

MADAMBA, P. S.; DRISCOLL, R. H.; BUCKLE, K. A. The thin-layer drying characteristics of garlic slices. Journal of Food Engineering, London, v. 29, n. 1, p. 7597, 1996.

MENDONÇA, R. C. et al. Flora vascular do Cerrado. In: SANO, S. M.; ALMEIDA, S. P. de (Eds.). Cerrado: ambiente e flora. Planaltina, DF: Embrapa-CPAC, 1998. p. 289-556.

MESQUITA, J. B.; ANDRADE, E. T.; CORRÊA, P. C. Modelos matemáticos e curvas de umidade de equilíbrio de sementes de jacarandá-da-bahia, angico-vermelho e óleo-copaíba. Cerne, Lavras, v. 7, n. 2, p. 12-21, 2001.

MOHAPATRA, D.; RAO, P. S. A thin layer drying model of parboiled wheat. Journal of Food Engineering, London, v. 66, n. 4, p. 513-518, 2005.

NAVES, R. N. Espécies frutíferas dos Cerrados de Goiás: caracterização e influências do clima e dos solos. 1999. 202 f. Tese (Doutorado em Agronomia) - Escola de Agronomia e Engenharia de Alimentos, Universidade Federal de Goiás, Goiânia, 1999.

NUNES, L. de S. et al. Avaliação do comportamento higroscópico de amido de inhame. Revista Brasileira de Produtos Agroindustriais, Campina Grande, v. 11, n. 2, p. 149-158, 2009.

OLIVEIRA, D. L.; ROCHA, C. Alternativas sustentáveis para a merenda escolar com o uso de plantas do Cerrado, promovendo educação ambiental. Revista Eletrônica do Mestrado em Educação Ambiental, Rio Grande, v. 21, n. 1, p. 35-53, 2008.

OLIVEIRA, G. H. H. et al. Evaluation of thermodynamic properties using GAB model to describe the desorption process of cocoa beans. International Journal of Food Science and Technology, Oxford, v. 46, n. 10, p. 20772084, 2011.

OLIVEIRA, M. M. da et al. Estudo do equilíbrio higroscópico da casca de abacaxi. Tecnologia e Ciência Agropecuária, João Pessoa, v. 3, n. 3, p. 63-66, 2009. 
OLIVEIRA, M. M. et al. Isotermas de dessorção da casca do maracujá (Passiflora edulis Sims): determinação experimental e avaliação de modelos matemáticos. Ciência Rural, Santa Maria, v. 36, n. 5, p. 1624-1629, 2006.

PRADO, M. E. T. et al. Isotermas de sorção de tâmaras: determinação experimental e avaliação de modelos matemáticos. Ciência e Tecnologia de Alimentos, Campinas, v. 19, n. 3, p. 233-236, 1999.

SILVA, A. S. et al. Característica higroscópica e termodinâmica do coentro desidratado. Revista Ciência Agronômica, Fortaleza, v. 41, n. 2, p. 237-244, 2010.

SILVA, C. C.; DEVILLA, I. A. Higroscopia das folhas de louro (Lauro nobilis L.). 2005. Disponível em: <http://prp. urg.br/06v1/conteúdo/pesquisainiccien/eventos/sic2005/ arquivos/agrarias/higroscopia.pdf $>$. Acesso em: $08 \mathrm{fev}$. 2012 .

SILVA, D. B. et al. Frutas do Cerrado. Brasília, DF: Embrapa Informação Tecnológica, 2001.
SILVA, J. A. da et al. Frutas nativas dos Cerrados. Brasília, DF: Embrapa-CPAC, 1994.

SILVA, M. M. da et al. Demanda energética envolvida no processo de dessorção de umidade em polpa de manga. Revista Brasileira de Produtos Agroindustriais, Campina Grande, v. 4, n. 2, p. 107-117, 2002.

SIRIPATRAWAN, U.; JANTAWAT, P. Determination of moisture sorption isotherms of jasmine rice crackers using BET and GAB models. Food Science and Technology International, Madrid, v. 6, n. 12, p. 459-465, 2006.

VIEIRA, R. F. et al. Frutas nativas da região CentroOeste. Brasília, DF: Embrapa Recursos Genéticos e Biotecnologia, 2006.

WANG, N.; BRENNAN, J. G. Moisture sorption isotherm characteristics of potato at four temperatures. Journal of Food Engineering, London, v. 14, n. 4, p. 269-287, 1991. 